\title{
ATENCIÓN HUMANIZADA DEL PARTO DE ADOLESCENTES: ¿NORMA, DESEO O REALIDAD?
}

\author{
Álida Andrade Sampaio ${ }^{1}$, Ângela Regina de Vasconcelos Silva ${ }^{1}$, Escolástica Rejane \\ Ferreira Moura, $P h D^{2}$.
}

${ }^{1}$ Enfermera, Universidad de Fortaleza, Brasil. ${ }^{2}$ Enfermera, Universidad Federal de Ceará, Brasil.

\section{RESUMEN}

Estudio exploratorio descriptivo que tuvo por objetivo analizar la atención al parto bajo la mirada de embarazadas adolescentes, en la perspectiva de la humanización. Se realizó en un hospital del distrito de Fortaleza-CE, en el período de marzo/abril de 2003 por medio de encuesta semiestructurada aplicada a 30 adolescentes. Algunos aspectos de la dinámica de los servicios fueron observados e informaciones juzgadas importantes fueron registradas en un diario de campo. La mayoría de las embarazadas desconoce y/o confunde la categoría de los profesionales que la asiste, aspecto negativo para la verdadera representación social en cuanto a la identidad profesional de los mismos. Necesidades en el campo del apoyo emocional y de recibir informaciones fueron destacadas por las deponentes y las prácticas que son útiles y que deben ser enfatizadas en la atención del parto (categoría A de la Organización Mundial de Sanidad - OMS) aparecieron de manera no-sistemática e implementadas a veces.

\section{PALABRAS CLAVE: Humanización del parto, adolescencia, enfermería, Brasil}

\section{SUMMARY}

Exploratory descriptive study that had by objective to analyze the attention to childbirth under the glance of pregnant adolescents, in the perspective of humanization. It was made in a hospital of the district of Fortaleza-CE, in a period of time between March/April of 2003 by interviews semi-structured applied to 30 adolescents. Some aspects of the services' dynamics were observed and informations judged as important were registered in a daily field. Most of the pregnant women does not know and/or confuses the category of the professionals that take care of them, negative aspect for the true social representation as well as their professional identity. Necessities in field related to emotional support and to receive informations were outstanding by the deponents and the practices that are useful and must be encouraged by occasion of the attention to the childbirth (category A of the World-wide Organization of Health - WHO) appeared as the notsystematic way and implemented sometimes.

KEY WORD: Humanization of the childbirth, adolescence, nursing, Brazil 


\section{INTRODUCCIÓN}

La atención a la salud materno-infantil ha provocado cambios significativos en el perfil de morbilidad y mortalidad materna en Brasil y, particularmente, en el Estado de Ceará (región del nordeste de Brasil), especialmente en las dos últimas décadas, teniendo en cuenta la reducción de la tasa de mortalidad infantil (TMI) y de las causas de mortalidad materna (CMM). Pero, persisten dificultades relacionadas con la calidad de la atención prenatal y del parto. En Ceará, en 1987, la TMI era superior a 100 muertes de menores de un año por 1.000 nacidos vivos; llegándose a registrar más de 6.000 muertes infantiles en un año. Al final del año 2002, la Secretaría Estadual de Sanidad registró tasa de 25 muertes de menores de un año por 1.000 nacidos vivos, representando una reducción del $70 \%$. Con relación a las causas de mortalidad materna (CMM), en 1995 se registraron 92 muertes maternas por 100.000 nacidos vivos, reducción equivalente al 85,9\% para el año 2002 (1).

En este contexto, la Enfermería desarrolla una actuación específica y fundamental en la implementación de acciones para la salud de la mujer, con énfasis en la atención de la gestación, parto y puerperio. Los desafíos enfrentados están relacionados con la calidad y con la humanización del cuidado prestado. En lo que respecta a los recursos humanos, la principal preocupación ha sido con el sentido de ofrecer cursos y oportunidades para una educación continuada, pero, sin embargo, el enfoque es dado principalmente al conocimiento y menos de las habilidades, con prácticamente omisión del componente de la actitud y de la ética. En la humanización, el foco ha sido la atención acogedora y segura al paciente, respetando sus derechos, elecciones e individualidad.

Se puede constatar que el proceso natural y fisiológico del parto en el país viene siendo, en general, inhibido. La embarazada pierde su autonomía, siendo sometida a la conveniencia de los profesionales de la salud y de los hospitales, ocasionando un elevado índice de partos por cesárea o inducidos. Por ello, Brasil se destaca, desde hace 30 años, como uno de los campeones en cesáreas, superado sólo por Chile y algunos pocos países asiáticos, cuando los estudios epidemiológicos han demostrado que la cesárea aumenta el riesgo de muerte materna y neonatal entre tres a siete veces comparados con el parto vaginal (2).

Frederick Leboyer, obstetra francés, considerado el precursor de la humanización, en su libro "Por un nacimiento sin violencia", ya llamaba la atención de la sociedad para la forma como "los se- res humanos eran traídos al mundo", cuestionando la práctica de atención del parto (3). En Brasil, los primeros en trabajar en pro de la humanización del parto y nacimiento fue el Profesor Galba de Araújo, en Fortaleza, y el Dr. Moisés Paciornick, en Curitiba (4). A partir del año 2000, se percibe la humanización del parto como una necesidad que genera un movimiento de ámbito nacional, el Programa de Humanización en el Prenatal y Nacimiento (PHPN), proyectado por el Ministerio de Sanidad, con el compromiso de despertar en los profesionales de salud, una visión favorable al respecto a la naturaleza y fisiología del parto y nacimiento (2). La atención humanizada al parto posibilita, a las embarazadas, disfrutar de recursos para tornar el proceso de alumbramiento más participativo, agradable y seguro. Son medidas simples que viabilizan una calidad diferenciada en la atención prestada por los profesionales de sanidad del centro obstétrico.

Las tasas de embarazo en la adolescencia varían mucho de servicio en servicio, pero se estima que en torno al $20-25 \%$ del total de embarazadas son adolescentes, sugiriendo que, de cada cinco embarazadas, una sea de este grupo de edad (5). Un estudio realizado por Moura (6) constató en una Regional de Sanidad de Ceará que el $23,4 \%$ de las embarazadas catastradas en el sistema de sanidad tenían menos de 20 años, con pequeña variación entre los ocho municipios que componen la región, a excepción del municipio de Mulungu, en el cual el $59,3 \%$ de las embarazadas eran adolescentes. Por lo tanto, la demanda de embarazadas adolescentes en las maternidades es significativa y constituye un grupo poblacional con características orgánicas, funcionales, sociales y emocionales que exigen un cuidado diferenciado por parte del equipo que pone atención al parto. La adolescente embarazada vive dos roles a la vez: la construcción de una nueva imagen corporal, proveniente de los cambios físicos y psicológicos propios de la pubertad y el proceso de adaptación al ciclo embarazo-puerperio, que también implica una serie de cambios a nivel corporal, fisiológico, afectivo y relacional (7). La experiencia que la adolescente vive, en busca de su identidad personal hace que el ejercicio de la maternidad, puede significar tanto un proceso de desarrollo e integración de su identidad de mujer, reafirmando vivencias anteriores e identificando nuevas alternativas para sí, o, al contrario, puede permanecer en patrones infantiles de relaciones y de comportamientos.

Considerando la relevancia de la atención de enfermería al parto humanizado y su importancia para las madres y recién-nacidos, se decidió realizar este estudio, el cual tiene por objetivo analizar 
la atención al parto recibida por adolescentes, en la perspectiva de la humanización. Se resalta que la Organización Mundial de la Salud (OMS) clasifica las prácticas comunes en la conducción del parto normal en cuatro categorías, dependiendo de su utilidad y eficacia. La categoría " $A$ " envuelve prácticas que son útiles y benéficas para el trabajo de parto y parto, favoreciendo, tanto a la embarazada como al bebé, y por ello, deben ser estimuladas (8). Entre esas prácticas el estudio buscó analizar las siguientes: ofrecer a la mujer el máximo de informaciones y explicaciones; libertad de posición y movimiento durante el trabajo de parto; técnicas no-invasivas y no-farmacológicas de alivio del dolor durante el trabajo de parto, como masaje y técnicas de relajación; y contacto precoz piel a piel entre madre y bebé y el apoyo al inicio del amamantamiento materno en la primera hora del posparto.

\section{MATERIAL Y MÉTODO}

Estudio de tipo exploratorio-descriptivo, cuya conjetura básica fue describir los significados atribuidos por las adolescentes al analizar la atención recibida al momento del parto, delineada por patrones de humanización preconizados por la Organización Mundial de la Salud (OMS) y adoptados por el Ministerio de Sanidad de Brasil. El carácter descriptivo de una encuesta es atribuido al contacto del pesquisidor con los sujetos investigados, permitiendo el conocimiento de las características del grupo, problemas y valores; en cuanto al carácter exploratorio, el propio autor atribuye la posibilidad de levantar los conocimientos existentes acerca de la temática estudiada para el encadenamiento con nuevas variables (9).

La encuesta fue realizada en el Hospital del Distrito Gonzaga Mota, ubicado en área metropolitana de Fortaleza - Messejana, en el período marzo-abril de 2003. Es una unidad de sanidad de referencia secundaria para la atención obstétrica y neonatal que atiende embarazadas de alto riesgo, realizando cerca de 470 partos mensuales. Vale destacar que esta unidad participó del Proyecto Luz, una iniciativa de la SESA-CE en convenio con la Agencia de Cooperación Internacional de Japón (JICA), teniendo como meta la humanización de la atención del parto y nacimiento. El sector obstétrico dispone de 10 camas para preparto y parto (PP), unificados. Son camas que fueron adquiridas por el Proyecto Luz y que ofrecen múltiplas posibilidades de cambio de posición, transformándose, inclusive, en silla, permitiendo a la embarazada elegir la posición más conveniente para parir (recostada, en cuclillas y otras). La cama PP es más ancha que en lo normal y posee colchón de mayor densidad para garantizar más confort a la mujer (9).

Otros recursos son usados para reducir la incomodidad de la embarazada y favorecer la evolución del trabajo de parto, como el apoyo en pelotas y caballitos y baño con agua tibia. El ambiente es decorado, con color verde, rompiendo la rigidez del ambiente hospitalario.

Los datos fueron obtenidos a través de una encuesta semi-estructurada realizada junto a 30 embarazadas adolescentes, cuyos partos ocurrieron en el respectivo hospital. Algunos aspectos de la dinámica de los servicios fueron observados e informaciones importantes fueron registradas en un diario de campo. La elección de las participantes del estudio fue aleatoria y de acuerdo con su aceptación voluntaria para participar. Explicaciones acerca del trabajo, incluyendo los objetivos y el método de encuesta, fueron dadas a los sujetos, así como también la garantía del sigilo y la posibilidad de salir de la encuesta si fuera conveniente. La participación fue oficializada por la firma del término de consentimiento, de conformidad con la Resolución 196/96 del Consejo Nacional de Sanidad en encuestas sobre seres humanos (10). Las embarazadas fueron identificadas por las iniciales de sus nombres.

Después de la recolección de datos, se inició la organización de estos, a través de lecturas sucesivas, percepción de las convergencias y categorización, teniéndose en cuenta las prácticas que son útiles y que deben ser enfatizadas durante el parto, seleccionadas entre las que componen la Categoría "A" de la OMS y descritas en la introducción del estudio. Además, los resultados presentan la caracterización de las participantes de la encuesta y la dificultad de identificación que éstas tienen con relación a la categoría profesional de las que las atienden.

\section{RESULTADOS Y DISCUSIÓN}

Características de las adolescentes participantes del estudio. Entre las 30 adolescentes puérperas que participaron de la encuesta, $10(33,3 \%)$ tenían edad entre 15 y 16 años, y 20 (76,7\%) entre 17 y 19 años. Se pudo identificar un universo significativo de embarazos en el inicio de la adolescencia (entre 15 y 16 años), período en que el embarazo se torna más preocupante comparado con la adolescencia más tardía, arriba de 17 años.

En cuanto a la escolaridad, $16(53,3 \%)$ tenían la enseñanza fundamental incompleta (1er grado) y $4(13,3 \%)$ completa; $8(26,7 \%)$ estaban cursando enseñanza media ( $2^{\circ}$ grado), y $2(6,7 \%)$ la habían 
concluido. Se percibe que la escolaridad de la mayoría de estas adolescentes sufrió algún tipo de interrupción a lo largo de los años, una vez que, a los 14 años se espera que hayan concluido la enseñanza fundamental; $y$ a los 17 la enseñanza media. El abandono y la evasión escolar es un problema identificado en el escenario educacional del país, el cual se está enfrentando, aunque tímidamente, por medio del incentivo financiero a las familias que mantienen sus hijos en la escuela, así como también invirtiendo en la red de enseñanza y calificación de profesores, de modo de tornarla más atractiva y útil para sus alumnos. Lo poco atractivo de las escuelas y la baja expectativa con relación a los proyectos de vida que algunos adolescentes visualizan para sí, contribuyen, asimismo, para esa quiebra en la secuencia escolar.

La frecuencia de adolescentes que vivían en unión consensual con el compañero fue $19(63,3 \%)$; casadas $5(16,7 \%)$ y solteras $6(20 \%)$. Se afirma que la situación conyugal insegura e inestable es condición desfavorable al embarazo, siendo apuntada como uno de los factores de riesgo obstétrico (11). De lo expuesto, se analiza que estar en unión consensual, casada o soltera, no significa estabilidad, seguridad, satisfacción o relación compartida, lo que hace sugerir que estudios de esta naturaleza estén vueltos para os los aspectos más subjetivos de la relación conyugal de las adolescentes, en el sentido de obtener datos más relevantes al debate.

Con relación a la ocupación, $21(70 \%)$ de las embarazadas se presentaron como domésticas, 6 (20\%) como estudiantes y $3(10 \%)$ sin ocupación. Estudios apuntan a que la maternidad en la adolescencia interfiere en el proceso de escolarización de las mujeres, notoriamente en aquellas que pertenecen a las clases sociales más bajas, una vez que los cuidados con el niño y la necesidad de buscar trabajo para contribuir con la renta familiar pasan a ser necesidades más emergentes (12). Con bajo nivel de escolaridad, tendrán dificultades para conseguir una inserción en el mercado de trabajo. Por otro lado, la escolaridad de la embarazada es un factor que merece atención en el campo de la maternidad, pues provee subsidios importantes para que las orientaciones suministradas durante el prenatal, parto y puerperio sean adecuadamente incorporadas y puestas en práctica en el cotidiano. De las entrevistadas $23(76,7 \%)$ eran primíparas y $7(23,3 \%)$ estaban en el segundo embarazo.

En cuanto a la religión, predominó la católica, con $21(70 \%)$ de la muestra, sin religión $5(16,7 \%)$ y $4(13,3 \%)$ que afirmaron ser protestantes. La religiosidad es un aspecto fundamental a ser trabajado con las adolescentes. Identificar la religión del individuo es muy poco en detrimento a identificar como el propio individuo participa de la religión, cuál(es) grupo(s) frecuenta. Geraldo Majela Agnelo (13) afirma que los jóvenes tienen un contenido religioso que excede todo lo que almacena la humanidad y con ansias por un mundo fraterno y justo.

Conocimiento de las adolescentes sobre la categoría profesional que la atendió en el parto. Cuando eran indagadas sobre la categoría del profesional que la atendió en el parto, la mayoría $22(73,3 \%)$ informó haber sido el médico y la enfermera; $3(10 \%)$ se refirieron a la enfermera; $2(6,7 \%)$ al médico; 2 $(6,7 \%)$ al médico juntamente con la auxiliar de enfermería y $1(3,3 \%)$ citó el académico. En el transcurso de las entrevistas llamó la atención el elevado contingente de embarazadas que afirmaron ausencia de presentación por parte de las enfermeras, o sea, $20(66,7 \%)$. Ante el conocimiento empírico de que los pacientes, en general, no distinguen la categoría del profesional que le está prestando el cuidado, se constató que enfermeras son confundidas con médicas y con auxiliares de enfermería, lo que merece ser tomado por el enfermero como una actividad a ser introducida. Estudio tipo sondeo con 28 sujetos participantes de un proyecto de extensión universitaria identificó como principal respuesta sobre "quién es la enfermera" - la auxiliar del médico, resultado indeseable (14).

Otro estudio realizado en el contexto de la atención primaria de un área rural de Ceará constató en el universo de 120 usuarios que 115 (95,8\%) supieron reconocer la enfermera del equipo del PSF y sólo $5(4,2 \%)$ la confundió con la auxiliar de enfermería. Este resultado es importante para la categoría de los enfermeros, una vez que la representación social sobre el enfermero siempre fue permeable de equívocos en cuanto a la identidad profesional. Este resultado fue atribuido a la postura adoptada por la enfermera del área: se presenta por ocasión de sus actividades y aprovecha todas las oportunidades de contacto con los usuarios para explicar sobre "quién es el enfermero", utilizando la misma identificación en la placa de señalización del consultorio, en su vestuario (capote que usa durante la atención), en carteles para divulgación de actividades, así como también refuerza, junto al equipo de sanidad, la importancia de la construcción de una representación social verdadera por parte de la comunidad, en cuanto a la profesión del enfermero. Por lo tanto, esta postura sencilla, verdadera, y comprometida, a lo largo de los cinco años de actuación de la enfermera del área en estudio, hizo la diferencia (15).

Ofrecer a la mujer el máximo de informaciones y 
explicaciones. Sólo 12 (40\%) de las encuestas explicaban a la embarazada los procedimientos a ser realizados, con todo, todas explicaban lo que habían encontrado, después de su realización. El consentimiento de la usuaria sobre los procedimientos, técnicas y cuidados a que va a ser sometida es un derecho, así como un principio ético y moral a ser seguido por los profesionales y que, por lo tanto, debe estar presente en la propuesta de humanización de la atención. Esa ausencia de comunicación e interacción positiva puede ser perjudicial a las madres, tornándolas ansiosas y tensas, pues afecta lazos de afectividad y confianza entre enfermera y embarazada, comprometiendo la meta de informar, esclarecer y encorajar a la mujer, envolviéndola en las decisiones e iniciativas en el acto del parto. Otro aspecto a ese respecto, es que, dar la información es esencial para el auto-cuidado de la embarazada y el cuidado del concepto y niño, correspondiendo al enfermero comunicarse efectivamente con la embarazada en todos los momentos de contacto y prestación de cuidados (16).

Libertad de posición y movimiento durante el trabajo de parto. De acuerdo con Balaskas (17), las evidencias enseñan las ventajas del parto fisiológico, en la posición vertical o en cuclillas. El autor afirma que la superficie del área del canal de parto puede aumentar en hasta el 30\% cuando la mujer pasa de la posición acostada para la posición en cuclillas. En la posición supina, el peso del útero grávido reduce el flujo sanguíneo placentario por la compresión de la arteria aorta descendente y de la vena cava inferior. Estudios recientes también comprobaron ventajas que una embarazada tiene cuando camina, o hace variaciones de la posición erecta, durante el trabajo de parto, en detrimento de aquellas que se mantienen en el lecho. En este contexto, el estudio constató que 19 (63,3\%) de las embarazadas adoptaron la posición horizontal durante el parto, seguido de $10(33,3 \%)$ que parieron en la posición semi-cuclillas (en la cama) y sólo una $(3,3 \%)$ adoptó la posición lateral.

Técnicas no invasivas y no farmacológicas de alivio de los dolores del trabajo de parto. Uno de los elementos considerado relevante en el cuidar humanizado de las embarazadas es la utilización de métodos no-invasivos y no-farmacológicos de alivio de los dolores del parto. De las embarazadas entrevistadas, 19 (63,3\%) afirmaron haber recibido del equipo de enfermería, algún recurso para minimizar los dolores del parto. Los más usados fueron el baño con agua tibia, masajes y el caballito o balancito que consiste en estimular la embarazada a mover las caderas de un lado para el otro de forma circular para facilitar el encaje y rotación del feto en la pelvis, disminuyendo la duración del parto. Son técnicas que estimulan el aumento del flujo sanguíneo, mejorando la circulación cutánea y de los tejidos y removiendo los productos del cansancio. Sin embargo, $11(36,7 \%)$ de las embarazadas no recibieron cualquier medio de alivio para minimizar sus dolores; una paciente informó haberse negado a recibir cualquier método de alivio y otra resaltó que no hubo tiempo para realizarlo, pues llegó al servicio en expulsivo.

De cualquier forma, medidas alternativas para minimizar los dolores del parto no están siendo implementadas sistemáticamente en todas las embarazadas, ni todos los profesionales de sanidad los tienen adoptado como rutina en el trabajo de parto. Los testimonios, a seguir, confirman estas observaciones: "Hubo una hora en que la enfermera hizo masaje, me puso a bañarme y se quedaba conmigo, a mi lado. Yo andaba siempre que podía" (J.I.O.B.). "Primeramente, la enfermera me puso en el caballito, después fui a la pelota, entonces en el caballito ella hizo masaje. ¡Ah! También me bañé en agua tibia, y eso disminuye más los dolores" (A.M.P.D.N.). "No, pues yo no quise. Ellas hasta dijeron para que yo me quedara en la pelota; quisieron hacer masajes, pero yo no quise; sólo quería caminar; no conseguía parar de caminar" (R.P.S.). "No, pues cuando yo llegué, ella ya estaba naciendo" (I.F.A.).

Estímulo al primer contacto físico entre madre y bebé y al amamantamiento posparto. De acuerdo con el Ministerio de Salud, los principales objetivos de la atención inmediata del recién nacido son proporcionar condiciones óptimas que permitan ayudarlos en su adaptación a la vida extra-uterina y estar preparado para intervenir en aquellos casos que presenten condiciones patológicas que pongan en riesgo su vida. Para la atención del recién nacido normal, nada más debe secarse, calentar, evaluar y entregar a la madre para un contacto íntimo y precoz. Todos los procedimientos habituales como pesar, aplicar vitamina $\mathrm{K}$ y otros deben ser realizados después del contacto de la madre (2). De las entrevistadas, 27 (90\%) tuvieron contacto precoz con sus recién nacidos. Ello es bastante positivo para ambos, pues, de acuerdo con la OMS (1996), el contacto piel a piel precoz entre la madre y el bebé es muy importante psicológicamente, pues estimula madre y bebé a conocerse, iniciando el eslabón afectivo. Las madres que no se quedaron con el bebé, fue debido a alguna intercurrencia. Sólo 10 (33,3\%) de las adolescentes fueron estimuladas a iniciar el amamantamiento aún en la sala de parto. Entre las que no fueron estimuladas, 8 $(26,7 \%)$ expresaron que "el niño había sido lleva- 
do, pronto después del parto, para ser limpiados", sin ninguna explicación, como fue relatado en los siguientes testimonios: "Pronto nació pusieron encima de mí, le cortaron el cordón umbilical y después le llevaron para bañarse" (R.F.C.). "Ellos llevaron el niño y sólo me lo entregaron al día siguiente, por la mañana”.(N.W.L.S.). "Ni el médico ni otra persona ordenó" (R.B.P.). De acuerdo con el Ministerio de Salud, son diversas las ventajas que la práctica del amamantamiento materno ofrece para el crecimiento y desarrollo de lactantes y para la madre, niño y familia, en los ámbitos biológico y psicológico. Sin embargo, a lo largo de los años, varios obstáculos han surgido en oposición a esa práctica, siendo uno de los principales factores no estimulantes de ese acto, creencias, mitos y tabúes arraigados fuertemente en la sociedad, principalmente en el seno familiar (2). En ese contexto, el enfermero ejerce un significativo papel de educador en sanidad, desmitificando condiciones que amenazan la instalación y mantenimiento del amamantamiento materno exclusivo hasta los seis meses de vida del bebé, debiendo promoverlo desde la sala de parto.

Necesidades y expectativas de las embarazadas con relación a la atención recibida. Se puede constatar que la atención de buena asistencia para esas mujeres se limita a la presencia de alguien a su lado, durante el trabajo de parto y parto, dando el apoyo y la atención necesaria para mantener el equilibrio emocional, reduciendo, así, sus tensiones y miedos. De este modo, aparecen en los relatos siguientes algunos de esos resultados, como buena atención prestada o como atención inadecuada: "Ya está. Si yo supiera que era así yo habría venido aquí para que aquí naciera mi primer hijo. Uno tiene miedo de que las personas sean ignorantes, que no tengan paciencia, pero fue todo al contrario. Yo las vi bañando a las otras mujeres, poniéndolas en el caballito y en la pelota, pena que no hubo tiempo para que yo lo hiciera ello también. Pero está todo maravilloso." (L.F.A.). "No sé explicar, una parte fue ruin y otra fue buena. Hasta que rompió aguas, ellas no hicieron nada por mí, pero, después que rompió aguas se quedaron conmigo. Entonces, cuando el bebé nació ellas salieron y me dejaron sola y con la placenta. Tuve que llamar a una persona para sacar la placenta y limpiarme" (A.C.G.G.). La relación entre enfermero y embarazada es hecha con atención, cordialidad, respeto y resolución de los problemas, una vez que el equipo de enfermería debe estar atento para las necesidades de la embarazada y sus expectativas, dispuesto a orientar y responder sus cuestionamientos, al fin, implementando observación, solidaridad, apoyo y calor humano, continuamente: "Sería bueno que todos los hospitales hicieran de esa manera. Ya es un sufrimiento tener el bebé y aún tener una mala atención. A mí mucho me gustó, y espero que mejore más y más". (L.C.F., 19 años). "Me gustaría que ellas fuesen más comprensivas, se quedasen con nosotras todo el tiempo que sea necesario". (J.S.). Se puede percibir, a través de lo que cuentan las embarazadas, que sus exigencias y expectativas acerca del cuidado de enfermería son mínimas, pues sólo solicitan la presencia de un profesional que les dé atención, cariño, orientación y les trasmitan seguridad a partir de sus conocimientos. Las embarazadas desean que ello sea extensivo a toda la red de sanidad. Esa reivindicación de las adolescentes en la medida que es extremamente simple es extremamente efectiva a la evolución del trabajo de parto. Se sabe que, en las condiciones de inseguridad y miedo, todo el proceso fisiológico y natural de evolución del trabajo de parto es inhibido. Circunstancias de esta naturaleza podrán generar múltiples complicaciones, una vez que el ciclo vicioso "Dolor $\rightarrow$ Miedo $\rightarrow$ Tensión $\rightarrow$ Dolor" bloquea la evolución del proceso fisiológico natural del parto normal (18).

\section{CONCLUSIÓN}

El estudio demostró que el enfermero está perdiendo oportunidades para construir su identidad profesional junto a las embarazadas, cuando se resalta que el campo de la Obstetricia es vasto a la actuación de estos profesionales.

En relación a las necesidades y expectativas de las adolescentes acerca de la atención recibida, se identificó que éstas solicitan solamente satisfacer sus necesidades afectivas (cariño, atención, afecto y comprensión) y de desarrollo (aprendizaje), lo que pueden ser realizadas por la enfermera, percibiendo el cuidado de enfermería con enfoque holístico. En este contexto, las mujeres ampliaron sus sentimientos, deseando que esa iniciativa fuera generalizada y expandida a todos los servicios de sanidad. En ese caso, hay aún mucho a ser conquistado para alcanzar la meta pretendida con la atención humanizada del parto. Cambios son sugeridos partiendo de una nueva política a ser implementada en las maternidades, con profesionales sensibilizados y comprometidos con la cuestión de la mejoría de la calidad de la atención del parto y su humanización, promoviendo efectivamente una atención humanizada, para la autonomía de las embarazadas, sin sumisiones, ofreciendo el cuidado que las mujeres necesitan, esperan y sueñan. 


\section{BIBLIOGRAFÍA}

1. Secretaría de la Sanidad. La Sanidad en Ceará en grandes números: una evaluación de la sanidad y de las acciones implementadas por el Sistema Público Estadual 1995-2002. Fortaleza (CE): Secretaría de Sanidad; 2002.

2. Ministerio de la Sanidad, Secretaría de Políticas de Sanidad, Área Técnica de Sanidad de la Mujer. Parto, aborto y puerperio: atención humanizada a la mujer. Brasilia (DF): Ministerio de la Sanidad; 2003.

3. Leboyer F. Por un nacimiento sin violencia. Río de Janeiro (RJ), 1974.

4. Cordeiro SN, Sabatino H. La humanización del parto. En: Zugaib M, Tedesco JJ, Quayle J. Obstetricia Psicosomática. São Paulo (SP): Editorial Ateneu; 1997; 280-317.

5. Santos Júnior JD. Factores etiológicos relacionados al embarazo en la adolescencia: vulnerabilidad a la maternidad. En: Ministerio de la Sanidad, Secretaría de Políticas de Sanidad. Cuadernos Juventud Sanidad y Desarrollo. Brasilia (DF): MS/SPS, 1999; 223-9.

6. Moura ERF. Atención de enfermería en el prenatal en el contexto del Programa Sanidad de la Familia [disertación]. Fortaleza (CE): Universidad Federal de Ceará; 2001.

7. Kahhale EMP. Mecanismos psíquicos de la embarazada adolescente. En: Zugaib M, Tedesco JJ, Quayle J. Obstetricia Psicosomática. São Paulo (SP): Editorial Ateneu; 1997; 243-51.

8. Secretaría de Sanidad. Maternidad segura y parto seguro. Boletín Luz, № 3. Fortaleza: 12 de agosto, 1998.
9. Triviños ANS. Introducción a la encuesta en Ciencias Sociales. São Paulo: Atlas, 1993.

10. Consejo Nacional de Sanidad. Resolución 196/96. Brasilia (DF): Ministerio de la Sanidad; 2001.

11. Ministerio de la Sanidad, Secretaría de Políticas de Sanidad. Atención prenatal: manual técnico. $3^{\underline{a}}$ ed. Brasilia (DF): Ministerio de la Sanidad; 2000.

12. Brienza AM apud Melo LS, Gomes FA, Almeida AM. Conocimiento de embarazadas acerca de las señales y síntomas del inicio del trabajo de parto. Revista Enfermería UERJ 2002, 10:176-181.

13. Majella Agnelo G. Juventud y religiosidad. En: Ministerio de Sanidad, Secretaría de Políticas de Sanidad. Cuadernos Juventud Sanidad y Desarrollo. Brasilia (DF): MS/SPS 1999; 57-62.

14. Stacciarini JM, Andraus LMS, Esperidião E, Nakatani AK. ¿Quién es el enfermero? Revista Electrónica de Enfermería, Goiania 1999; 1(1). Disponible en: http:// www.fen.ufg.br/revista.

15. Vasconcelos AC, Moura ERF. Percepción del rol desempeñado por la enfermera de un PSF, según la óptica del usuario. Revista RENE 2003;4:9-16.

16. Burroughs A apud Rodrigues PD, Montesuma FG, Silva RM. El significado del parto y de la atención de enfermería: visión de mujeres en puerperio inmediato. Revista RENE 2001,2:101-6.

17. Balaskas J. Parto activo: guía práctica para el parto natural. 2a ed. São Paulo (SP): Ground; 1993.

18. Secretaría de Sanidad. Maternidad segura y parto seguro. Boletín Luz, №8. Fortaleza (CE): 23 de enero, 1999. 